\title{
Polygraphies. Les frontières du littéraire, éds. Jean-Paul Dufiet et Elisabeth Nardout-Lafarge
}

\section{Roberta Sapino}

\section{(2) OpenEdition}

1 Journals

\section{Edizione digitale}

URL: http://journals.openedition.org/studifrancesi/5477

DOI: $10.4000 /$ studifrancesi. 5477

ISSN: 2421-5856

\section{Editore}

Rosenberg \& Sellier

\section{Edizione cartacea}

Data di pubblicazione: 1 dicembre 2016

Paginazione: 584-585

ISSN: 0039-2944

\section{Notizia bibliografica digitale}

Roberta Sapino, «Polygraphies. Les frontières du littéraire, éds. Jean-Paul Dufiet et Elisabeth Nardout-

Lafarge », Studi Francesi [Online], 180 (LX | III) | 2016, online dal 01 janvier 2017, consultato il 18 septembre 2020. URL : http://journals.openedition.org/studifrancesi/5477 ; DOI : https://doi.org/ 10.4000/studifrancesi.5477

Questo documento è stato generato automaticamente il 18 settembre 2020.

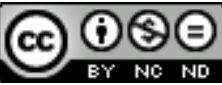

Studi Francesi è distribuita con Licenza Creative Commons Attribuzione - Non commerciale - Non opere derivate 4.0 Internazionale. 


\title{
Polygraphies. Les frontières $d u$ littéraire, éds. Jean-Paul Dufiet et Elisabeth Nardout-Lafarge
}

\author{
Roberta Sapino
}

\section{NOTIZIA}

Polygraphies. Les frontières du littéraire, sous la direction de Jean-Paul DUFIET et Elisabeth NARDOUT-LAFARGE, Paris, Classiques Garnier, 2015, 375 pp.

1 Scopo del volume è contribuire alla rivalutazione e al reinserimento del concetto di poligrafia all'interno del discorso critico, dimostrandone la produttività analitica ed esplicativa tanto nello studio della figura dell'autore quanto nella comprensione delle singole opere. L'introduzione storica e teorica, firmata dai curatori Jean-Paul DUFIET e Elisabeth NARDOUT-LAFARGE (Avant-propos. Réflexions sur une notion critique: la poligraphie, pp.7-25), pone basi solide per una riflessione che considera un corpus cronologicamente molto esteso (dal Medioevo all'età contemporanea), e che comprende esempi di poligrafia sia interna sia esterna, sollecitando nozioni affini quali l'intertestualità e il genere letterario.

Dalla nascita del romanzo, avvenuta sotto il segno della pluralità di voci e del rimaneggiamento di materiali preesistenti (Francis GINGRAS, La polygraphie à l'œuvre. Du recueil médiéval au roman recyclé, pp. 29-40), alle operazioni culturali di appropriazione e trasferimento di testi classici compiute nel Rinascimento tanto da eruditi di professione quanto da figure amatoriali non meno importanti (Sergio CAPPELlo, Polygraphie et polygraphes au $\mathrm{xVI}^{\mathrm{e}}$ siècle, pp.41-64), fino alla dimensione poligrafica assunta dal romanzo periodico settecentesco sotto la spinta di autori come Marivaux, Prévost e Mouhy (Ugo DIONNE, L'art d'écrire à saute-mouton. Roman périodique et polygraphie au XVIII siècle, pp.65-84), la prima sezione traccia un percorso diacronico piuttosto rappresentativo de «Le sources historiques et littéraires de la polygraphie». 
3 Un salto cronologico importante conduce alla seconda sezione, dedicata ai rapporti complessi tra «Polygraphie et généricité» e inaugurata da un saggio sulla fortuna dello scrittore-giornalista nel Québec del Novecento, e in particolare sul valore di esercizio di libertà letteraria che la poligrafia assume nell'opera di Berthelot Brunet (Michel BIRON, L'écrivain journaliste au Québec. L'exemple de Berthelot Brunet, pp. 87-102). Con François DUMONT si ritorna in Francia per addentrarsi tra Poème et carnet chez Philippe Jaccottet, forme diverse nelle quali risuona la stessa ambizione di "justesse" (pp. 103-114), mentre Catherine Douzou si concentra sull'integrazione di forme e tematiche romanzesche nel teatro di Jean-Luc Lagarce, inserendo la propria analisi all'interno di una più vasta presentazione delle pratiche di ibridazione nel teatro contemporaneo (Jean-Luc Lagarce, entre roman et théâtre. Un polygraphe postmoderne, pp. 115-133). Klaus-Dieter ERTLER di sofferma infine su una figura che chiama Le professeur-romancier ou le polygraphe québécois contemporain e della quale Catherine Mavrikakis, Eric Dupont, Julie Mazzieri e Régine Robin costituiscono esempi rappresentativi in virtù della loro capacità di incarnare, ed esprimere attraverso la scrittura, una certa permeabilità tra il sistema accademico e il mondo letterario (pp. 135-154).

4 La sezione successiva si sviluppa invece come una rassegna di diverse forme di «Écritures polygraphiques». Il contributo di Pierre GLAUDES dimostra la fecondità della pratica poligrafica di Mirabeau: non segno di corruzione della scrittura letteraria, ma strumento artistico e ideologico in cui comicità e senso critico danno vita a una forma ibrida discendente dalla satira menippea (Mirabeau polygraphe, pp. 157-172). Jean-Paul DUFIET analizza l'opera della scrittrice di origine polacca Anna Langfus, e osserva come la commistione tra teatro e letteratura testimoniale su cui si fonda il "drame éthique" Le Lépreux consenta all'autrice di ripensare e trasmettere l'esperienza vissuta (Théâtre et témoignage dans l'écriture de la Shoah. "Le Lépreux" d'Anna Langfus, pp. 173-197). Poligrafia, schizofrenia e plagio sono i concetti sui quali Marylin RANDALL fonda la sua analisi di Trou de Mémoire, dalla quale emerge come la poligrafia praticata da Hubert Aquin costituisca non un mezzo d'espressione di sé, ma un luogo di dissolvimento dell'io a vantaggio del processo di creazione (Polygraphie schizophrène et plagiat chez Hubert Aquin. Le cas de "Trou de Mémoire", pp. 199-213). Valeria SPERTI studia la figura di Nancy Huston entre essai et fiction: dialogando con le scritture di Romain Gary e Samuel Beckett attraverso forme di ibridità tanto generiche quanto linguistiche, Huston situa la sua pratica poligrafica alle frontiere del testo, sfumando progressivamente le frontiere tra saggio e finzione (pp. 215-236). Dopo avere illustrato i termini del dibattito suscitato dal saggio di Monique LaRue L'Arpenteur et le Navigateur, il contributo di MartineEmmanuelle LAPOINTE propone una lettura di L'oeil de Marquise condotta attraverso la lente del "roman de filiation" teorizzato da Viart, e volta a rilevare il desiderio dell'autrice di inscrivere la propria soggettività nell'avvenire collettivo della Storia (Le passage de l'essai au roman dans l'œuvre de Monique LaRue. "L'œil de Marquise", réécriture de "L'Arpenteur et le Navigateur", pp. 237-249).

5 Il volume si conclude con alcuni esempi significativi di interazione tra «Polygraphie et intermédialité» nel mondo contemporaneo, dai quali emerge la straordinaria fecondità delle pratiche poligrafiche basate sull'incontro tra forme espressive diverse. Sperimentazioni di poligrafia tanto esterna quanto interna consentono a Antonio D'Alfonso di far risuonare lo spaesamento di un'identità deterritorializzata all'interno di un'opera intermediale complessa (Alessandra FERRARO, L'écriture polygraphique d'Antonio D'Alfonso, pp.253-273); Claude Gauvreau, con un movimento opposto, 
contrasta la dispersione poligrafica della sua opera lavorando quasi ossessivamente al progetto di un'opera omnia che sarà poi pubblicata postuma (Gilles LAPOINTE, Claude Gauvreau, polygraphe inquiet. Réflexion sur l'esthétique moderniste des "Euvres créatrices complètes", pp. 275-293); alcuni momenti di incontro fra le pratiche creative di Françoise Sullivan e Denise Desautels danno origine a felici commistioni di scrittura e danza, musica e arti plastiche (Ginette michaud, Chorégraphies, disent-elle: ce qui (se) passe entre les arts. Quelques remarques au sujet de trois échanges entre François Sullivan et Denise Desautels, pp. 295-324). Il contributo di Andrea SCHINCARIOL chiude il percorso rivalutando in ottica 2.0 alcune problematiche emerse negli articoli precedenti; lo studio di opere di François Bon (Tumultes) e Anne Savelli (Fenêtres / Open Space e Franck) lo porta a suggerire che il nuovo ruolo assunto dal lettore all'interno del dispositivo intermediale costituisca una possibile chiave di comprensione della poligrafia nell'era digitale (Polygraphie 2.0. Le texte à l'ère de la reproductibilité numérique, pp. 325-343). 